\title{
Rechte an Daten
}

Daniel Hürlimann / Herbert Zech *

Daten sind zu einem Rohstoff geworden, der neue Wertschöpfungsketten ermöglicht. Dessen ungeachtet ist die Frage, welche Rechte an Daten bestehen, bisher zumindest in der Schweiz - nur wenig bearbeitet worden. Ziel des vorliegenden Beitrags ist es, mit Blick auf die Rechtslage in der Schweiz eine erste Übersicht zu Rechten, die an Daten bestehen können, zu geben. Daran anschliessend wird auch die Frage aufgeworfen, ob an der heutigen Rechtslage etwas geändert werden soll.

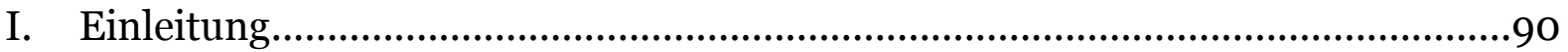

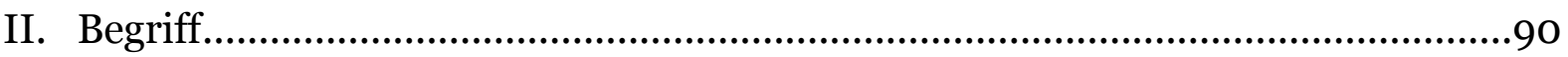

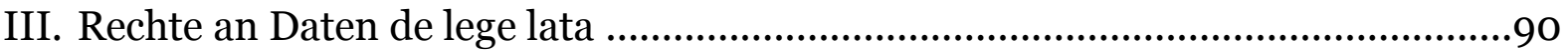

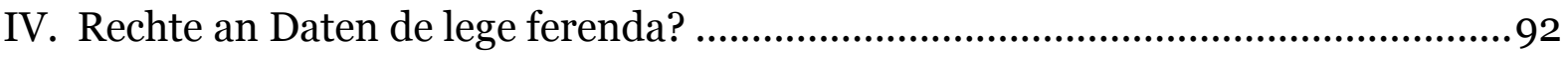

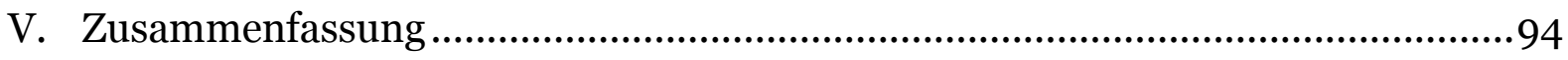

Zitiervorschlag: Daniel Hürlimann / Herber Zech, Rechte an Daten, in: sui-generis 2016, S. 89

URL: $\quad$ sui-generis.ch/27

DOI: $\quad$ https://doi.org/10.21257/sg.27

* Prof. Dr. iur. Daniel Hürlimann ist Assistenzprofessor für Informationsrecht und Direktor der Forschungsstelle für Informationsrecht an der Universität St.Gallen.

Prof. Dr. iur. Dipl.-Biol. Herbert Zech ist Professor für Life Sciences-Recht und Immaterialgüterrecht an der Universität Basel.

Dieses Werk ist lizenziert unter einer Creative Commons Namensnennung - Weitergabe unter gleichen Bedingungen 4.0 International Lizenz. 


\section{Einleitung}

1 Man mag es kaum mehr hören: Daten sind das Öl des 21. Jahrhunderts. Der Vergleich mag in Bezug auf die wirtschaftliche Relevanz eine gewisse Gültigkeit haben. Gleichzeitig ist er aber in verschiedener Hinsicht unsinnig: Öl kann man nutzen und dann ist es in der Regel weg. Öl kann man kaufen und dann gehört es niemand anderem. Öl kann man aus einer bestimmten Quelle fördern und damit verhindern, dass es jemand anderes tut. Demgegenüber kann man Daten nutzen, ohne dass sie danach weg wären. Man kann Daten kaufen, ohne dass damit ausgeschlossen wäre, dass ein anderer diese Daten auch kauft. Und man kann Daten sammeln, ohne damit zu verhindern, dass sonst jemand die gleichen Daten auch sammelt.

2 Kurz (oder ökonomisch gesprochen): Öl ist rivalisierend, Daten sind es nicht. Nicht rivalisierende Güter können von mehreren Personen gleichzeitig verwendet werden, ohne dass eine Verwendung die andere beeinträchtigt. Öl ist darüber hinaus auch ausschliessbar, der Eigentümer kann also kontrollieren, wer den Rohstoff nutzt und wer nicht. Die Frage der Ausschliessbarkeit ist bei Daten weniger eindeutig: Kann die Verwendung von Daten durch Dritte ausgeschlossen werden? Falls ja, auf welche Rechtsgrundlage könnte sich der Ausschluss stützen? Der vorliegende Beitrag versucht, erste Antworten auf diese Fragen zu geben.

\section{Begriff}

3 Daten sind gemäss Duden «(durch Beobachtungen, Messungen, statistische Erhebungen u.a. gewonnene) [Zahlen] werte, (auf Beobachtungen, Messungen, statistischen Erhebungen u.a. beruhende) Angaben, formulierbare Befunde» sowie «elektronisch gespeicherte Zeichen, Angaben, Informationen»1. Daten können also einerseits auf der Bedeutungsebene (semantische Information) und andererseits auf der Zeichenebene (syntaktische Information) abgegrenzt werden ${ }^{2}$. Ersteres ist der Ansatz des Datenschutzrechts, das Daten als Angaben, die sich auf eine bestimmte oder bestimmbare Person beziehen, definiert3. Grenzt man Daten auf der Zeichenebene ab, könnte damit eine Menge von Einsen und Nullen als Rechtsobjekt geschützt werden4.

\section{Rechte an Daten de lege lata}

4 Wer die faktische Herrschaft über Daten hat, kann die Verwendung derselben durch Dritte ganz einfach dadurch verhindern, dass er die Daten nicht weitergibt. Dies funktioniert natürlich nur, solange die Daten geheim sind. Daneben besteht immer auch die Möglichkeit, die Datennutzung vertraglich zu regeln, ohne dass die Rechtsnatur von Daten geklärt sein muss. Unabhängig davon soll nachfolgend untersucht werden, welche Rechte an Daten nach heute geltendem Recht bestehen.

5 Im geltenden Recht werden Daten in erster Linie durch das Datenschutzgesetz erfasst. Das Gesetz bezieht sich jedoch nur auf Personendaten und definiert diese in Art. 3 lit. a DSG als «alle Angaben, die sich auf eine bestimmte oder be-

\footnotetext{
Duden Online zum Begriff «Daten».

2 Herbert Zech, «Industrie 4.0» - Rechtsrahmen für eine Datenwirtschaft im digitalen Binnenmarkt, GRUR 2015, S. $1151 \mathrm{ff}$.

3 Art. 3 lit. a DSG.

4 Zech (Fn. 2), S. 1153.
} 
stimmbare Person beziehen». Das Datenschutzgesetz schützt nicht Daten als solche, stattdessen «bezweckt [es] den Schutz der Persönlichkeit und der Grundrechte von Personen, über die Daten bearbeitet werden» (Art. 1 DSG). Dies zeigt sich z.B. an der Regel, wonach Personendaten dann nicht ins Ausland bekannt gegeben werden dürfen, «wenn dadurch die Persönlichkeit der betroffenen Personen schwerwiegend gefährdet würde» (Art. 6 Abs. 1 DSG). Während für Datenbearbeitungen durch Private immer das Bundesrecht massgebend ist, muss bei Datenbearbeitungen durch Behörden je nach Stufe derselben das eidgenössische oder das jeweilige kantonale Datenschutzgesetz berücksichtigt werden. Die Datenschutzgesetze regeln die Voraussetzungen, unter denen eine Bearbeitung von Personendaten zulässig ist. Sie enthalten jedoch keine Regeln zur Frage, welche Rechte an Daten bestehen.

Mit der Zuweisung von Ausschliesslichkeitsrechten an unkörperlichen und deswegen nicht-rivalisierend nutzbaren Gütern beschäftigt sich bereits seit Langem das Immaterialgüterrecht. Aus dem Kanon der bestehenden Immaterialgüterrechte kommt für Daten allenfalls das Urheberrecht in Betracht. Das Urheberrecht schützt Werke, d.h. geistige Schöpfungen der Literatur und Kunst, die individuellen Charakter haben (Art. 2 Abs. 1 URG). Art. 2 Abs. 2 URG nennt beispielhaft verschiedene Werkarten, darunter Sprachwerke, akustische Werke und Werke der bildenden Kunst. All diese Werke können auch digital entstehen oder digitalisiert werden, damit werden sie zu Daten und diese Daten sind urheberrechtlich geschützt. Bei der reinen Ansammlung von Daten liegt aber in al- ler Regel keine geistige Schöpfung vor und diese Daten sind vom Urheberrecht nicht geschützt.

7 Neben den Immaterialgüterrechten bietet das Lauterkeitsrecht einen gewissen Schutz bestimmter Leistungen bzw. Leistungsergebnisse vor Nachahmung. Das Lauterkeitsrecht verbietet insbesondere die Übernahme oder Verwertung eines marktreifen Arbeitsergebnisses, wenn dies durch technische Reproduktionsverfahren und ohne angemessenen eigenen Aufwand erfolgt (Art. 5 lit. c UWG). 5 Davon können auch Daten erfasst sein, wenn sie Teil des marktreifen Arbeitsergebnisses sind. Ansonsten kennt aber auch das Lauterkeitsrecht keinen eigenständigen Schutz von Daten.

8 Nachdem bis vor Kurzem Einigkeit darüber zu bestehen schien, dass das Sachenrecht nicht auf Daten anwendbar ist, hat ECKERT unter dem Titel «Digitale Daten als Wirtschaftsgut: digitale Daten als Sache» diese These unlängst wieder hervorgeholt ${ }^{6}$. Das Zivilgesetzbuch widmet sich in über 300 Artikeln dem Sachenrecht, ohne aber zu definieren, was eine Sache ist. Die Lehre umschreibt die Sache als unpersönlichen, körperlichen und abgegrenzten Gegenstand, wobei ein Teil der Lehre darüber hinaus verlangt, dass die Sache der menschlichen Herrschaft unterworfen werden können muss7. Die-

$5 \longdiv { \text { Eine Regelung, die ursprünglich für den Schutz } }$ von Software eingeführt würde, die zwischenzeitlich aber auch durch Urheberrecht geschützt wird.

6 Martin Eckert, Digitale Daten als Wirtschaftsgut: digitale Daten als Sache, SJZ 2016, S. 245; Martin Eckert, Digitale Daten als Wirtschaftsgut: Besitz und Eigentum an digitalen Sachen, SJZ 2016, S. 265.

7 Liver, Schweizerisches Privatrecht (SPR) V/1, Basel 1977, S. 11; BSK-Wiegand, Vor Art. 641 N 5; BK-Meier-Hayoz, Art. 641 N 10. Dies ergibt sich 
se Streitfrage braucht hier nicht vertieft zu werden, zumal Einigkeit darüber besteht, dass die Körperlichkeit zur Definition gehört. Daten sind nicht körperlich und somit keine Sachen ${ }^{8}$. Ergänzend sei hinzugefügt, dass Eigentum auch wegen seiner zeitlich unbegrenzten Dauer nur rivalisierende Nutzungen erfassen bzw. zuweisen kann.

9 Ferner bestehen in einzelnen Bereichen Spezialbestimmungen betreffend Daten, so zum Beispiel im Geoinformationsgesetz und im Humanforschungsrecht. Neu und dem schweizerischen Recht bis vor Kurzem fremd ist hier insbesondere die Quellenangabepflicht für Geobasisdaten in Art. 50 der Geodatenverordnung9. Das Humanforschungsgesetz enthält neben einem Kapitel zu Transparenz und Datenschutz (Art. 56 ff. HFG) auch ein Kapitel über die Weiterverwendung von biologischem Material und gesundheitsbezogenen Personendaten für die Forschung (Art. 32 ff. HFG).

10 Als Zwischenfazit kann festgehalten werden, dass das geltende Recht für die meisten Daten keine Regelung und damit auch keine Zuordnung zu einem Rechtssubjekt vorsieht. Diese Rechtslage ist im europäischen Vergleich einzigartig, weil in der EU zumindest ein Datenbankschutz existiert ${ }^{10}$.

systematisch aus Art. 713 ZGB: «Gegenstand des Fahrniseigentums sind die ihrer Natur nach beweglichen körperlichen Sachen sowie die Naturkräfte, die der rechtlichen Herrschaft unterworfen werden können und nicht zu den Grundstücken gehören.»

8 So auch Rolf H. Weber / Christian Laux / Dominic Oertly, Datenpolitik als Rechtsthema, Zürich 2016, S. 54.

9 Verordnung über Geoinformation (Geoinformationsverordnung, GeoIV) vom 21. Mai 2008 (SR 510.620).

10 Richtlinie 96/9/EG des Europäischen Parlaments

\section{Rechte an Daten de lege ferenda?}

11 Da de lege lata keine eigenständigen Rechte an Daten existieren, soll nachfolgend kurz die Frage aufgegriffen werden, ob diese Rechtslage verändert werden soll bzw. welche Gründe dafür und welche dagegen sprechen.

12 Für die Schaffung eines neuen Datenrechts spricht die Tatsache, dass Daten einen Wert haben und deshalb ein wirtschaftliches Interesse an einem neuen Recht an Daten als «Rohstoff»11 bestehen könnte. Diese Situation ist jedoch nicht neu und ein Blick auf die heute schon bestehende Datenwirtschaft zeigt, dass es durchaus erfolgreiche Unternehmen gibt, deren Geschäftsmodell sich massgeblich auf Daten stützt. Mit Google basiert eines der mächtigsten Unternehmen überhaupt zu einem Grossteil auf Daten. Ein neues Recht an Daten würde es Unternehmen wie diesem wohl erschweren, an die notwendigen Daten zu kommen.

13 Ein Recht an Daten könnte also auch als Schutz des Individuums ausgestaltet werden, welches dann entscheiden könnte, wer welche Daten sammeln und verwalten darf. Ein solches Datenrecht hätte unzweifelhaft einschneidende Konsequenzen für zahlreiche Unternehmen

und des Rates vom 11. März 1996 über den rechtlichen Schutz von Datenbanken (Datenbankrichtlinie). Der Schutz erfasst allerdings auch nur die Datenbank insgesamt und nicht die einzelnen Daten, die in ihr enthalten sind.

11 Viktor Mayer-Schönberger/Kenneth Cukier, Big Data - A Revolution That Will Transform How We Live, Work and Think, 2013, S. 5. Es entstehen also neue Wertschöpfungsketten, bei denen die Erzeugung, Sammlung und Analyse von Daten als getrennte Stufen identifiziert werden können, die auch von unterschiedlichen Akteuren durchgeführt werden können. Als Erzeuger kommen dabei nicht nur Unternehmen, sondern auch Verbraucher in Betracht. 
und müsste schon deshalb von Beginn an international eingeführt werden. Würde ein Staat wie die Schweiz ohne internationale Abstimmung ein solches Recht einführen, bestünde die Gefahr, dass Unternehmen, deren Geschäftsmodelle auf Daten basieren, ihre Geschäftstätigkeit in andere Staaten verlagern würden. Nichtsdestotrotz haben die staatspolitischen Kommissionen von National- und Ständerat einer parlamentarischen Initiative Folge gegeben, welche ein Eigentumsrecht an Daten in der Verfassung verankern will ${ }^{12}$. Die Kommission des Nationalrats wird somit eine Gesetzesvorlage ausarbeiten, die anschliessend dem Gesamtrat unterbreitet werden wird ${ }^{13}$.

Während ein neues Recht an Daten auf den ersten Blick interessant erscheint, muss berücksichtigt werden, dass ein unilateral eingeführtes Recht letztlich dem Wirtschaftsstandort Schweiz grossen Schaden zufügen könnte. Nichtsdestotrotz gibt es gute Gründe für eine Stärkung der Rechtsposition des Individuums. In diesem Zusammenhang verdient insbesondere die Idee eines Rechts auf Kopie eine genauere Betrachtung. Auch dazu gibt es bereits einen politischen Vorstoss, dem eine Mehrheit des Nationalrats zugestimmt hat. Es handelt sich um ein Postulat, das vom Bundesrat die Erarbeitung eines Berichts zur Frage, inwiefern der Einzelne und die Volkswirtschaft von der Weiterverwendung von personenbezogenen Daten profitieren könnten, verlangt. Dabei soll er insbe-

12 Parlamentarische Initiative Fathi Derder, eingereicht am 20. Juni 2014: Schutz der digitalen Identität von Bürgerinnen und Bürgern (Geschäftsnr. 14.434).

13 Art. 111 des Bundesgesetzes über die Bundesversammlung (Parlamentsgesetz, ParlG, SR 171.10) sondere ein «Recht auf Kopie» für den Einzelnen prüfen ${ }^{14}$. Der Bundesrat hat in seiner Stellungnahme zu diesem Postulat festgehalten, dass er die verlangte Prüfung im Rahmen der Revision des Datenschutzgesetzes vornehmen werde.

15 Bei der Revision des Datenschutzgesetzes $^{15}$ wird der Schweiz einmal mehr nichts anderes übrig bleiben, als die Rechtsentwicklungen der EU zu übernehmen. Zu gross wären die wirtschaftlichen Nachteile, sollten in der Schweiz andere Regeln gelten als in den umliegenden EU-Staaten. Somit ist davon auszugehen, dass das im Postulat Derder vorgeschlagene Recht auf Kopie ohnehin im Rahmen des Nachvollzugs der EUDatenschutz-Grundverordnung (EUDSGVO'16) auch in das schweizerische Datenschutzgesetz Eingang finden wird.

16 Von zentraler Bedeutung sind die Regelungen zur Datenportabilität in der Datenschutz-Grundverordnung. Art. 15 Abs. 3 Satz 1 EU-DSGVO lautet: «Der Verantwortliche ${ }^{17}$ stellt eine Kopie der perso-

14 Postulat Fathi Derder, eingereicht am 25. September 2015: Recht auf Nutzung der persönlichen Daten. Recht auf Kopie (Geschäftsnr. 15.4045).

15 Der Bundesrat hat das EJPD im April 2015 beauftragt, ihm bis spätestens Ende August 2016 einen Vorentwurf für eine Revision des DSG zu unterbreiten (Medienmitteilung des Bundesrates vom 1. April 2015). Diesem Auftrag ist das EJPD bisher nicht nachgekommen.

16 Verordnung (EU) 2016/679 des Europäischen Parlaments und des Rates vom 27. April 2016 zum Schutz natürlicher Personen bei der Verarbeitung personenbezogener Daten, zum freien Datenverkehr und zur Aufhebung der Richtlinie 95/46/EG (Datenschutz-Grundverordnung).

17 Gemäss Art. 4 Ziff. 7 EU-DSGVO bezeichnet der Ausdruck «Verantwortlicher» «die natürliche oder juristische Person, Behörde, Einrichtung oder andere Stelle, die allein oder gemeinsam mit anderen über die Zwecke und Mittel der Verarbeitung von personenbezogenen Daten entscheidet; 
nenbezogenen Daten, die Gegenstand der Verarbeitung sind, zur Verfügung.» In Art. 15 Abs. 4 ist mit Verweis auf Abs. 3 explizit vom «Recht auf Erhalt einer Kopie» die Rede. Art. 20 EU-DSGVO enthält darüber hinaus ein Recht auf Datenübertragbarkeit. Die direkte Übermittlung von einem Anbieter zu einem anderen soll nach Art. 20 Abs. 2 EU-DSGVO verlangt werden können, «soweit dies technisch machbar ist». Unabhängig davon sieht Abs. 1 vor, dass die betroffene Person das Recht hat, "die sie betreffenden personenbezogenen Daten, die sie einem Verantwortlichen bereitgestellt hat, in einem strukturierten, gängigen und maschinenlesbaren Format zu erhalten». Mit Blick auf diese Regelungen in der EU ist davon auszugehen, dass das Recht auf Kopie auch in der Schweiz im Zuge der anstehenden DSG-Revision eingeführt wird.

Bei den Regelungen zur Datenportabilität handelt es sich eher um einen Anspruch auf Zugang zu Daten, der sich auf personenbezogene Daten beschränkt und dem Betroffenen zusteht. Ob darüber hinaus ein echtes Vermögensrecht an Daten eingeführt werden soll, das unabhängig von einem Personenbezug besteht, wird derzeit auch auf europäischer Ebene heftig diskutiert. ${ }^{18}$ Eine Einführung in

sind die Zwecke und Mittel dieser Verarbeitung durch das Unionsrecht oder das Recht der Mitgliedstaaten vorgegeben, so kann der Verantwortliche beziehungsweise können die bestimmten Kriterien seiner Benennung nach dem Unionsrecht oder dem Recht der Mitgliedstaaten vorgesehen werden;».

18 Eine Stellungnahme der Kommission wurde in der EU-Roadmap für November 2016 angekündigt. In diesem Zusammenhang sei auch auf die Erwähnung des freien Datenverkehrs auf dem EU-Binnenmarkt als fünfte Freiheit im Programm des slowakischen EU-Ratsvorsitzes, S. 9, hingewiesen. der Schweiz unabhängig vom Ausgang dieser Diskussion ist keinesfalls ratsam.

18 Die Gretchenfrage bei einem eigentumsartigen Recht an Daten wäre, wem dieses zustehen soll. Weder der Betroffene (Datenschutzrecht, setzt Personenbezug voraus) noch der «Inhaber» oder «Besitzer» (Sacheigentum, setzt faktische Herrschaft voraus) kommen in Betracht. Denkbar wäre, auf den wirtschaftlich verantwortlichen Erzeuger oder den «Skribenten» (technischer «Ersteller» der Daten ${ }^{19}$ ) abzustellen. Ob das aber ökonomisch sinnvoll ist, müsste erst empirisch untersucht werden. Weitere Fragen, die geklärt werden müssten, sind, welche Befugnisse dem Rechtinhaber ausschliesslich zugewiesen sein sollen (und ob sie sich auf semantische oder syntaktische Daten beziehen), welche Schranken für diese Zuweisung vorgesehen sein müssen, wie lange ein solches Recht dauern darf und ob zusätzliche Entstehungsvoraussetzungen wie etwa eine Registrierung zu fordern sind. Auch die Übertragung im Rechtsverkehr und das Verhältnis zu anderen Rechten wie dem Datenschutzrecht müssen genau bedacht werden. All diese Fragen sind noch ungeklärt.

\section{Zusammenfassung}

19 De lege lata existieren in der Schweiz keine eigenständigen Rechte an Daten. Datenschutz-, Urheber- und Lauterkeitsrecht schützten Personen, Werke und bestimmte Leistungen. Daten werden dementsprechend nur dann erfasst, wenn es sich um Personendaten, um digitalisierte bzw. digitale Werke oder um das Ergeb-

19 Thomas Hoeren, Dateneigentum, in: MMR 2013, 486 ff., S. 487. 
nis einer ausnahmsweise eigenständig durch das UWG geschützten Leistung handelt. Das Sachenrecht ist wegen der fehlenden Körperlichkeit von vornherein nicht auf Daten anwendbar.

20 Die Frage, ob an dieser Rechtslage etwas geändert werden soll, wird im Rahmen des Nachvollzugs der EU-DatenschutzGrundverordnung und möglicher weiterer EU-Rechtsakte in das schweizerische Recht zu diskutieren sein. Von der Einführung eines Eigentumsrechts an Daten ohne eingehende Überprüfung der Folgen ist dringend abzuraten, während die Idee eines Rechts auf Kopie das Potential hat, dem Individuum zumindest den $\mathrm{Zu}$ gang zu seinen Daten zu sichern. 